\title{
Machine Learning Applied to Neuroreabilitation: A Systematic Mapping
}

\author{
Eder Manoel de SANTANA1, Felipe Roque MARTINS', Flávia Gonçalves FERNANDES ${ }^{2}$ and Eduardo \\ Lázaro Martins NAVES ${ }^{1}$ \\ ${ }^{1}$ Universidade Federal de Uberlândia, Uberlândia-MG, Brasil \\ ${ }^{2}$ Instituto Federal de Educação, Ciência e Tecnologia Goiano, Campos Belos - GO, Brasil \\ profedersantana@gmail.com
}

\begin{abstract}
Machine learning and recommendation systems are tools used to improve the search indices of the most relevant items in large amounts of data that can be applied in the health area. To present a systematic mapping in the area of neurorehabilitation that uses machine learning. Analyze the references of the work carried out involving the theme on the application of machine learning in the area of neurorehabilitation. Search for studies enrolled in databases through logical operators for the selection of peer-reviewed journal articles. In addition, it was verified that the application of the systematic mapping in the elaboration of the bibliographic review allows to identify the main gaps for the development of new research, and to direct to the main publications related to the study. Therefore, it is necessary to promote this area of research to offer this public access to the techniques of neuro-rehabilitation as a form of treatment, acquisition of knowledge, motivation or even inclusion. In this way, it will be possible to obtain a greater maturity in the obtained results and, thus, to promote a systematization in the use of neurorehabilitation in the promotion of the well-being of these people.
\end{abstract}

Index Terms - Bioinformatics, Machine learning, Learning systems, Support vector machines, Computer applications.

\section{INTRODUCTION}

$\mathrm{T}$ here are now numerous therapies available to improve the conditions of people who have had, for example, limited movement, impaired communication or even their cognitive potential. In addition, there is a growing understanding that the approach should be multidisciplinary and individualized for each patient.

Neurorehabilitation is defined as a complex medical process that aims to assist in the recovery of a nervous system injury and to minimize and/or compensate for any functional changes resulting from such injuries. In this perspective, neurorehabilitation is a new concept for those who live with permanent pictures or sequelae, such as those occurring after stroke, meningitis, brain or spinal traumas, or even in situations that are caused by genetic diseases [1].

In addition, machine learning and referral systems are tools used to improve the search indexes for the most relevant items in large amounts of data, which can be widely applied in the healthcare, including hemotherapy and neural rehabilitation institutions and activities, for example.

Machine learning is a subfield of computer science that has evolved from the study of pattern recognition and the theory of computational learning in artificial intelligence. The objective of machine learning is the development of computational techniques on learning as well as the construction of systems capable of gaining knowledge automatically [2].

A learning system is a computer program that makes decisions based on accumulated experiences through the successful solution of previous problems. It is a powerful tool for automatic acquisition of knowledge. However, there is no single algorithm that presents better performance for all problems [3].

Induction is the form of logical inference that allows conclusions to be drawn from a set of examples. In it, a concept is learned by inductive inference on the examples presented (having caution in choosing such examples). Meanwhile, in deduction, humans use deductive reasoning to deduce new information from logically related information [2]

The algorithms use improved statistical analyzes on the data they receive, resulting in more accurate responses and predictions. Machine learning allows software applications to become more intelligent and predictive, without having to program them frequently for them to learn [4].

Machine learning technology can be categorized as: supervised or unsupervised. When algorithms are supervised, this means that a human controls the input and output of desired data and provides feedback on the accuracy of predictions during training. When complete, the algorithm applies what has been learned to a new data set [3].

In the unsupervised category, the algorithms need not be trained with desired result data. They use an iterative approach called deep learning. Unsupervised algorithms are used for more complex processing tasks [3].

A system that needs to perform well in a dynamic environment often requires adaptive or evolutionary solutions. Evolutionary systems try to solve problems by accumulating knowledge about the problem and using this knowledge to generate acceptable solutions. These problems are found in the areas of complex system configuration, task allocation, route selection, and other optimization and machine learning issues. Here are some examples of systems that can use machine learning: control of dynamic systems; induction and optimization of rules bases; definition of neural architectures; 
simulation of biological systems; interactive image evolution; musical composition [4].

The possibility of predicting machine learning can detect frauds, network security threats and perform predictive maintenance routines, and can help in the diagnosis of diseases, for example.

In this context, the objective of this work is to verify the panorama of related research on the application of machine learning in neurorehabilitation, presenting a systemic study about what has been published for such a field.

The purpose of this systematic mapping is to analyze the references of the work carried out involving the thematic about the application of machine learning in the area of neurorehabilitation. Moreover, to verify the rise of this line of research in the actuality.

\section{METHODS}

In this work, a systematic mapping was performed according to a proposed methodology by [5], consisting of a research of studies in databases through logical operators for the selection of articles, from the selection of keywords or key expressions.

The recent databases for this work were IEEE Xplore [6], Science Direct [7] and PubMed [8], these databases being available at the Federal University of Uberlândia. It is necessary to emphasize that only peer-reviewed journal articles were analyzed.

The logic expressions used for the research in the bases were: "learning machine" and "neurorehabilitation". Such strings were chosen to search works that involve machine learning in the healthcare. Filters were then selected to reduce the scope of the search. For example, for language (English and Portuguese), type of publication (peer-reviewed journal article) and year of publication (2008 to December 2018, constituting 10 years of search space). Once the filters were applied in each database, the titles were read in order to receive the data according to a selected logical expression. In addition, it was noted possible replications between the databases, and the articles that passed the inclusion criteria had all duplicates removed.

Finally, a final step in the selection of articles consisted in the targeting of applications that involved machine learning in the healthcare. For that, the selection was done through the reading and the analysis of the titles and abstract, in order to exclude the works that were not directly related to the subject being studied. In this sense, works that dealt with different themes were discarded, such as works that deal with machine learning aimed at application in other areas, for example.

\section{RESUlTS E DISCUSSION}

After searching the databases, the results were organized in the form of tables and graphs with the purpose of presenting them in a more practical way. Table I shows the total results obtained in the mapping, considering the aforementioned keywords and the December 2018 deadline.
Thus, through a systematic mapping, a database was created with 33 (thirty-three) database references for the combination of the strings "learning machine" AND "neurorehabilitation".

The evolution of the annual publication of the selected

\begin{tabular}{lcll}
\hline \multicolumn{1}{c}{ Databases } & Nmber of papers & pap \\
& ers \\
& Initial & $\begin{array}{c}\text { With adhering } \\
\text { title }\end{array}$ & $\begin{array}{l}\text { in } \\
\text { the } \\
\end{array}$ \\
\hline \hline PubMed & 102 & 24 & inter \\
IEEE Xplore & 22 & 8 & nati \\
Science Direct & 44 & 9 & sear \\
Total & $\mathbf{1 6 8}$ & $\mathbf{4 1}$ & ch \\
can & & & can
\end{tabular}

be seen in Figure 1, where it is possible to notice that the articles involving the subject addressed in this work were published in recent years. In this way, it is observed that the subject is recent and with great possibilities of fields to be explored.

\section{Number of papers per year of publication}

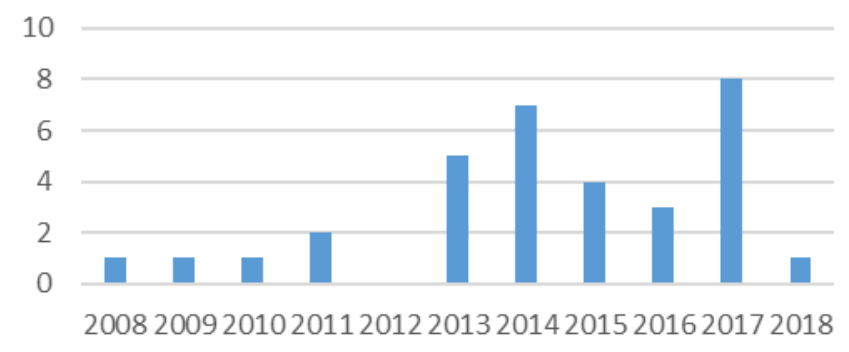

Fig. 1. Number of papers per year of publication.

Figure 2 shows the number of journal articles published by country. The papers published are from different countries, such as New Zealand, Italy, Japan, France, China, United States, Spain, United Kingdom, Germany, Iran, Australia, Colombia, Argentina, Denmark, Canada and Switzerland. In this way, it is verified that the majority of the works found that encompass the use of the machine learning in the neurorehabilitation come from developed countries.

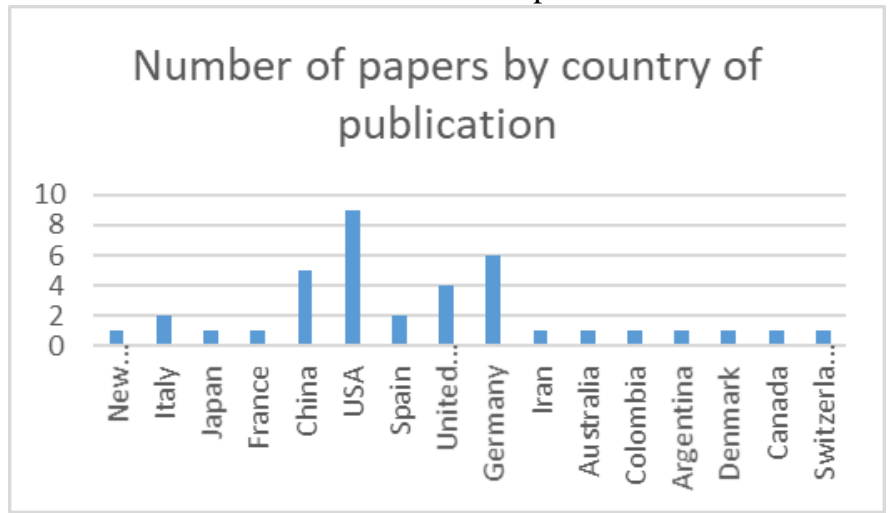

Fig. 2. Number of papers by country of publication. 
After reading the articles selected, some main topics were identified for the grouping of the results obtained, as can be observed in Figure 3, which are: stroke; stroke and spinal injury; neurodegenerative disease; spinal cord injury; Parkinson's disease and quadriplegia. Thus, through the visualization of the graph, it is noticed that the majority of the works found are directed to the neurorehabilitation of stroke.

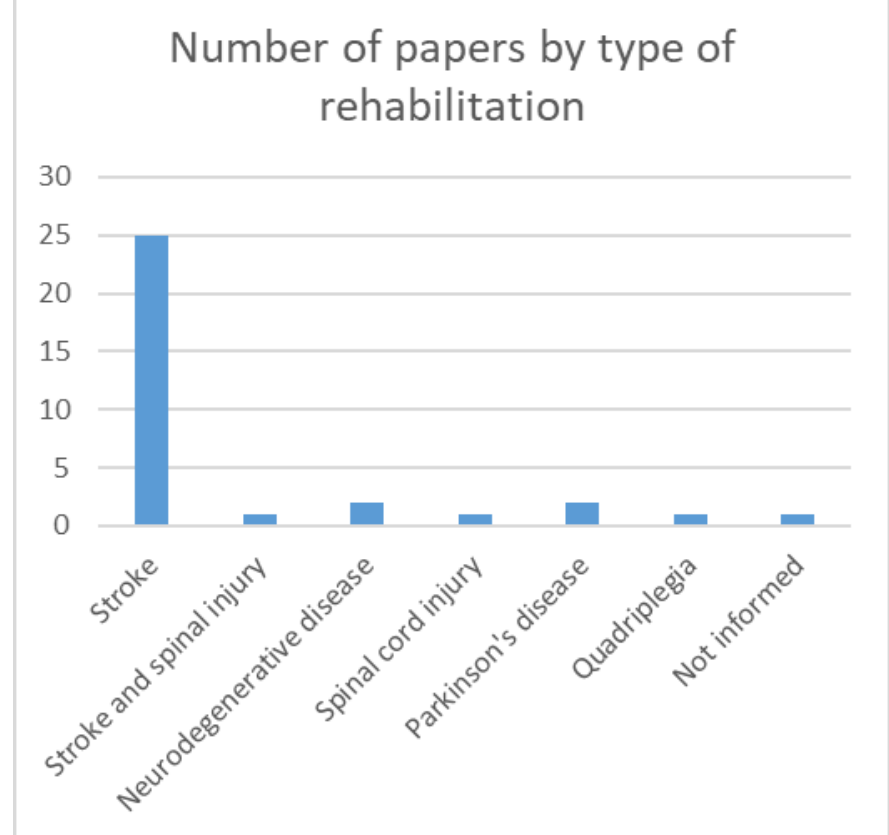

Fig. 3. Number of papers by type of rehabilitation.

Figure 4 shows the computational strategies used in the selected works that address the use of machine learning in neurorehabilitation, namely: Spiking Neural Network (SNN), Common Spatial Pattern (CSP), Support Vector Machine (SVM), Markov Decision Process MDP), Random Forest Algorithm (RFA), Maximum Entropy Method (MEM), Backpropagation Neural Network (BNN), Artificial Neural Network (ANN).

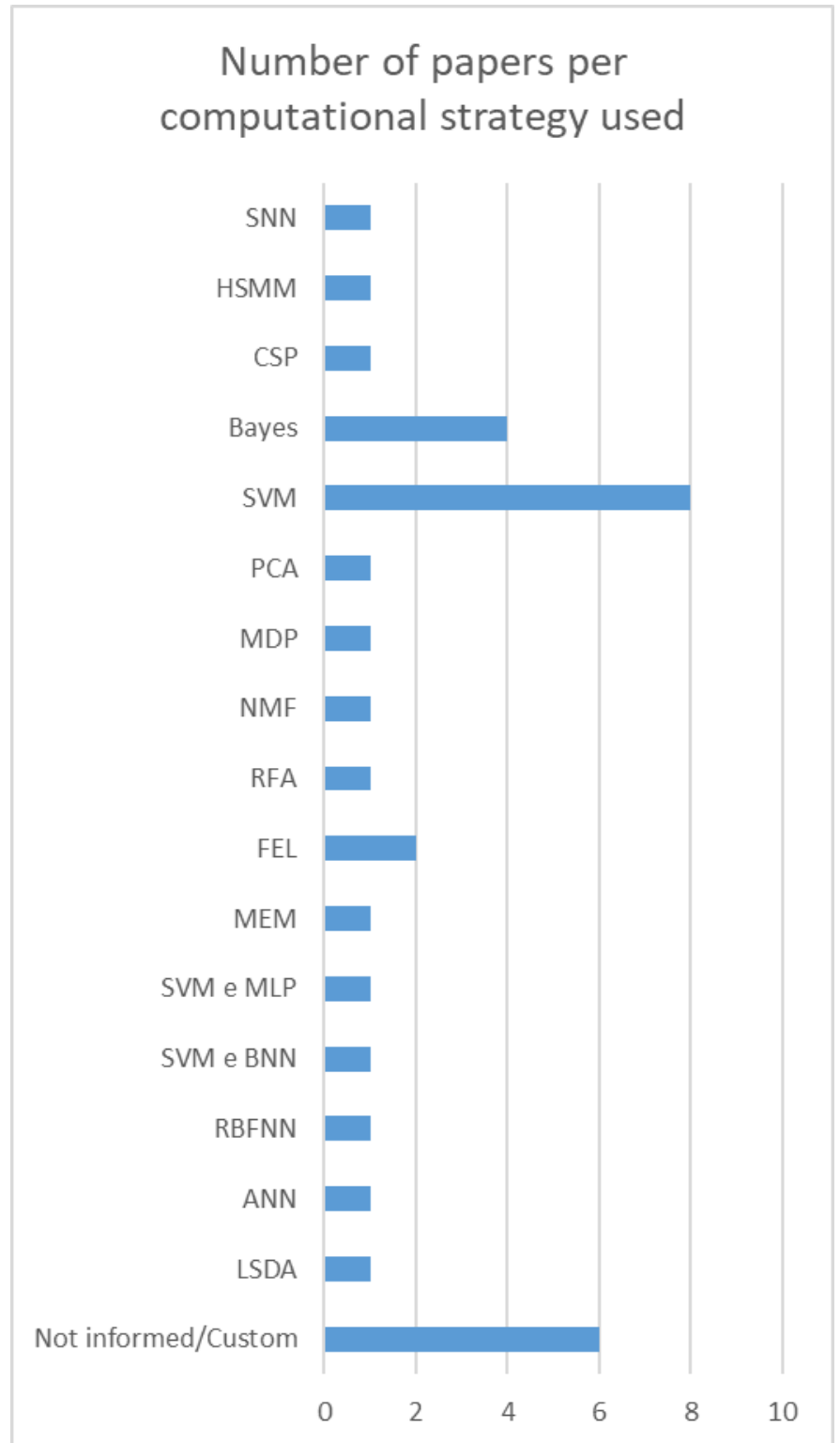

Fig. 4. Number of papers per computational strategy used.

The above strategies can be defined as: Spiking Neural Network (SNN), which is a network capable of imitating the cerebral cortex with learning ability, allowing modeling and achieving more realism in a neural simulation. SNN is part of the third generation of neural networks and is able to extract information through sensors and make high-level decisions [9].

Common Spatial Pattern (CSP), which is a technique that calculates filters that maximize the variance ratio between brain activities, and is, however, vulnerable to noise and overfitting problem [10].

Support Vector Machine (SVM) is a technique used for data analysis and pattern recognition for acting in the classification and regression analysis. The SVM acts as a non-probabilistic binary linear classifier that for each given input predicts between two possible classes to which the data belong. Based on sets of training examples divided into two categories, the 
SVM constructs a model for dividing the data between the two categories [11].

Markov Decision Process (MDP) is a model where the results are partially under control of a decision maker based on high-quality probabilistic calculations with meta-analytic data [12]. Random Forest Algorithm (RFA) searches for relevant important characteristics during classification, being a technique that seeks to achieve a maximum classification accuracy rate [13]. Maximum Entropy Method (MEM) is a method from statistical physics as a theoretical tool to predict the equilibrium states of thermal systems to design observations distribution functions [14].

Backpropagation Neural Network (BNN) uses an error output to change the weight values that are connected to the neurons in a hidden layer in the reverse direction until the ideal weight values that minimize the function of the network performance are reached [15]. Artificial Neural Network (ANN) is a technique that simulates the functioning of a neuron being widely used in machine training for task execution or decision making based on a classification pattern [16].

From the graph of Figure 4, it is observed that the Support Vector Machine SVM was the most used computational technique in the works found, since it is a newer tool and has several advantages that make its use attractive [10]:

- Good generalizability: classifiers generated by an SVM in general achieve good generalization results. The generalizability of a classifier is measured by its efficiency in classifying data that does not belong to the set used in its training. In the generation of predictors by SVMs, therefore, overfitting is avoided, in which the predictor becomes very specialized in the training set, obtaining low performance when confronted with new standards.

- Robustness in large dimensions: SVMs are robust against large objects, such as images. Commonly there is the occurrence of overfitting in the classifiers generated by other intelligent methods on these data types.

- Convexity of the objective function: the application of the SVMs implies the optimization of a quadratic function, which has only a global minimum. This is an advantage over, for example, Artificial Neural Networks, where there is the presence of local minimums in the objective function to be minimized.

- Well-defined theory: SVMs have a well-established theoretical basis within Mathematics and Statistics.

Figure 5 shows the types of input signal used in the selected works that address the use of machine learning in neurorehabilitation, which are: electroencephalography (EEG), kinematics, electromyography (EMG), EMG and kinematics, electrocorticography (ECoG) and biosensors.

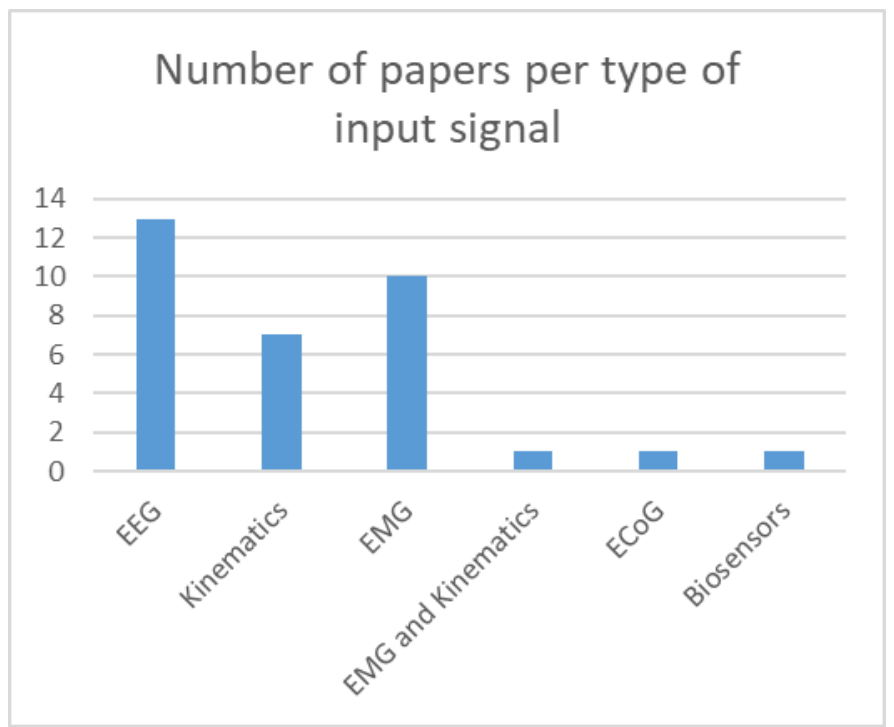

Fig. 5. Number of papers per type of input signal.

The signals obtained in the selected works can be defined as: Electroencephalography (EEG), which performs the analysis of brain electrical activity through the use of electrodes overlapping the scalp. This type of signal can be obtained in individuals of any age since it is present from birth. Electromyography (EMG) is a method used to diagnose and prognosis the lesions of the peripheral nervous system in order to locate an injury, provide information about the pathophysiology of the lesions, evaluate the degree of impairment as well as the temporal course of the lesion. The signal is obtained through stimulation of the sensory, peripheral and motor nerves through an electric current [17]. The electrocorticography $(\mathrm{ECoG})$ exam is used to measure electrical brain signals through electrodes subduedly implanted in the brain surface. Studies point to substantial information obtained in certain frequency bands in the use of this technique. Most of the signal is captured at gamma level, in the range of 70-100 $\mathrm{Hz}$ [18]. Biosensors are devices used to measure chemical or biological reactions by generating signals proportional to the concentration of analyte in the reaction. They are widely used for disease monitoring, detection of pollutants, disease-causing microorganisms, among others [17].

According to the graph of Figure 5, it can be observed that the electroencephalography (EEG) signal was the most observed in the studies found, since it is a less invasive procedure and with good signal quality, making it possible to extract substantial information. Another impact factor for the adoption of this type of examination is due to the fact that it does not have any contraindications and a minimum duration of twenty minutes.

Figure 6 shows the applications used in the selected works that address the use of machine learning in neurorehabilitation, namely: event prediction, score, motor imagery, assistance, analysis and training. 


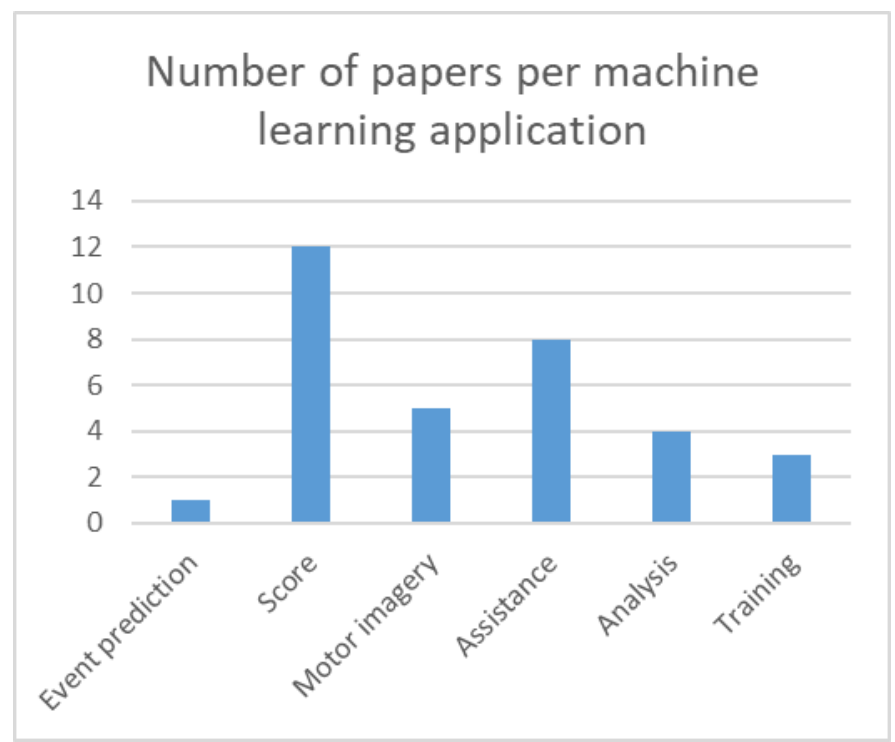

Fig. 6. Number of papers per machine learning application.

The applications classified as "event prediction" are intended to predict the probability of occurrence of stroke. The "score" category is designated when the application is used to evaluate an exercise or system. The category "motor imagery" is used to conceptualize the exercise of creating a "command image" in the brain, i.e., mentalizing the movement of a limb, for example, using EEG to read the user's intention to move. The "assistance" applications are algorithms used to aid in the performance or execution of user movements. The "analysis" is classified as the category that analyzes the data available in the literature or from experiments performed with users. In addition, the category "training" is adopted when mentioning works that perform the training of user or patient movements.

Figure 7 shows the percentage of papers that experimented with participants. Thus, it was observed that the majority $(76 \%$ of the studies) performed this procedure. This demonstrates the need to carry out tests to prove the results and effectiveness of the applications, as well as assist in the realization of improvements and maintenance of the systems.

\section{Percentage of articles that have experimented with participants}

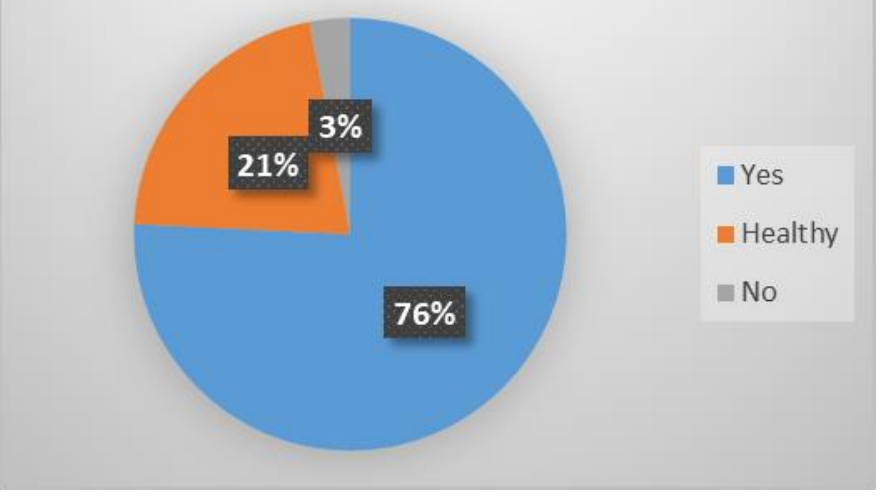

Fig. 7. Percentage of articles that have experimented with participants.

\section{CONCLUSIONS}

Through the mapping performed, it was possible to verify that there was a growth in the study of the application of machine learning in the area of neurorehabilitation, since it is a new and very efficient technique.

Thus, it should be noted that the application of systematic mapping allows identifying the main gaps for the development of new research. In addition, it addresses the main publications linked to the study.

In addition, it has been observed that machine learning is a field of computational intelligence research that studies the development of methods capable of extracting concepts (knowledge) from data samples. In general, the various machine learning algorithms are used in order to generate classifiers for a set of examples. By classification, the process of assigning, to a given information, the label of the class to which it belongs. Therefore, machine learning techniques are employed in the induction of a classifier, which must be (ideally) able to predict the class of any instances of the domain in which it was trained.

Due to what was presented in this study, there is a growing interest in researching and publishing in this area related to machine learning in the area of neurorehabilitation, seeking to assist in the neurological rehabilitation of people with various diseases.

Therefore, the need to promote this area of research to offer this public with neurological pathologies access to the techniques of neurorehabilitation as a form of treatment, acquisition of knowledge, motivation, entertainment or even by inclusion. In this way, it will be possible to obtain a greater maturity in the obtained results and, thus, to promote a systematization in the use of neurorehabilitation in the aid of the promotion of the well-being of these people.

\section{REFERENCES}

[1] Daur, L. Neurological rehabilitation. 6th Edition. Mosby; 2012.

[2] Russell, S.; Norvig P. "Artificial intelligence: a modern approach". 3rd Edition. Upper Saddle River: Prentice Hall, 2010.

[3] Alpaydin, E. "Introduction to machine learning". 2nd Edition. Editora MIT Press, 2010.

[4] Marsland, S. "Machine Learning: an algorithmic perspective". Editora Chapman \& Hall, 2009.

[5] Bailey, J. et al. "Evidence relating to object-oriented software design: A survey". First International Symposium on Em-pirical Software Engineering and Measurement. Computer Society; 2007.

[6] _. "IEEE Xplore Digital Library". 2019. Disponível em: <https://ieeexplore.ieee.org>. Acesso em: 18 jun. 2019.

[7] _. "Science Direct". 2019. Disponível em: <http://www.sciencedirect.com/>. Acesso em: 18 jun. 2019.

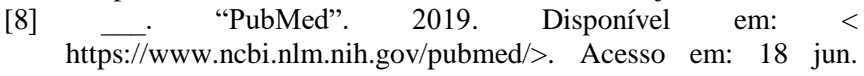
2019.

[9] Cheng, L. et al. "Biologically inspired spiking Neural Network for autonomous locomotion control of snake-like robots". International Journal of Biosensors \& Bioelectronics. 2017; 2(5):1-3. 
[10] Meisheri, H. "Multiclass common spatial pattern for EEG based brain computer interface with adaptive learning classifier". Pattern Recognition Letters, Computer Science. 2018.

[11] Frasson, C.; Kostopoulos, G. "Brain function assessment in learning". First International Conference. 2017.

[12] Choi, S. E.; Brandeau, M. L.; Basu, S. "Dynamic treatment selection and modification for personalised blood pressure therapy using a Markov decision process model: a costeffectiveness analysis". BMJ Journals. 2017.

[13] Nkambou, R.; Azevedo, R.; Vassileva, J. "Intelligent tutoring systems". 14th International Conference. 2018.

[14] Han, C.; Lan, J.; Fu, Q.; Chen, X. "Valence quark distributions and structure function of the free neutron from Maximum Entropy Method". High Energy Physics. Phenomenology. 2018.

[15] Puspita, J. W.; Jaya, A. I.; Gunadharma, S. "Classification of epileptiform and wicket spike of EEG pattern using Backpropagation Neural Network". American Institute of Physics. 2017.

[16] Santos, M. A. O.; Figueredo, J. D. S.; Bezerra, L. S.; Magalhães, F. N. O. "Neuronal plasticity mechanisms induced by brainmachine interfaces: connecting brain to artificial neural network". Revista de Medicina e Saúde de Brasília. 2017.

[17] Bhalla, N.; Jolly, P.; Formisano, N.; Estrela, P. "Introduction to biosensors". Department of Electronic and Electrical Engineering. University of Bath, 2016.

[18] Atashzar SF. et al. "A computational-model-based study of supervised haptics-enabled therapist-in-the-loop training for upper-limb poststroke robotic rehabilitation". IEEE/ASME Transactions on Mechatronics. 2018.

[19] Badesa FJ et al. "Auto-adaptive robot-aided therapy using machine learning techniques". Computer Methods and Programs in Biomedicine. 2013.

[20] Bhagat NA. "Detecting movement intent from scalp EEG in a novel upper limb robotic rehabilitation system for stroke". Conf Proc IEEE Eng Med Biol Soc. 2014; 2014:4127-4130.

[21] Cesqui B et al. "EMG-based pattern recognition approach in post stroke robot-aided rehabilitation: a feasibility study'. Journal of NeuroEngineering and Rehabilitation. 2013; 10:75.

[22] Capecci M et al. 'A Hidden Semi-Markov Model based approach for rehabilitation exercise assessment'. In: Journal of Biomedical Informatics. 2017; 78(1):1-11.

[23] Coste A et al. 'Preliminary developments towards closed-loop FES-assistance of posture and gait'. International Federation of Automatic Control Papers OnLine. 2015; 48(20):333-337.

[24] Darvishi S et al. 'Proprioceptive feedback facilitates motor imagery-related operant learning of sensorimotor ß-band modulation'. Frontiers in Neuroscience. 2017; 11(1).

[25] Del-Amaa AJ et al. 'Hybrid therapy of walking with Kinesis overground robot for persons with incomplete spinal cord injury: A feasibility study'. Robotics and Autonomous Systems. 2014.

[26] Dobkin BH et al. 'Reliability and validity of bilateral ankle accelerometer algorithms for activity recognition and walking speed after stroke'. Stroke. 2011; 42(8):2246-2250.

[27] Dutta A, Paulus W, Nitsche MA. 'Facilitating myoelectriccontrol with transcranial direct current stimulation: a preliminary study in healthy humans'. Journal of NeuroEngineering and Rehabilitation. 2014; 11(13).

[28] Gianfelici F. 'RBF-based technique for statistical demodulation of pathological tremor'. IEEE Transactions on Neural Networks and Learning Systems. 2013; 24(10).

[29] Gharabaghi A et al. 'Coupling brain-machine interfaces with cortical stimulation for brain-state dependent stimulation: enhancing motor cortex excitability for neurorehabilitation'. Frontiers in Human Neuroscience, 2014

[30] Griffith H, Biswas S. 'Home-based upper extremity rehabilitation support using a contactless ultrasonic sensor'. Conf Proc IEEE Eng Med Biol Soc. 2017; 2017:853-856.

[31] Hil NJ et al. 'Recording Human Electrocorticographic (ECoG) Signals for neuroscientific research and real-time functional cortical mapping'. Journal of Visualized Experiments. 2012.
[32] Kasabov $\mathrm{N}$ et al. 'Evolving spiking neural networks for personalised modelling, classification and prediction of spatiotemporal patterns with a case study on stroke'. Neurocomputing. 2014; 134(1):269-279.

[33] Lau H, Tong K, Zhu H. 'Support vector machine for classification of walking conditions of persons after stroke with dropped foot'. Human Movement Science. 2009; 28(1):504-514.

[34] Lin C et al. 'Discriminative manifold learning based detection of movement-related cortical potentials'. Transactions on Neural Systems and Rehabilitation Engineering. 2016.

[35] Liu D et al. 'Brain-actuated gait trainer with visual and proprioceptive feedback'. Journal Neural Eng. 2017.

[36] Liu Y et al. 'A tensor-based scheme for stroke patients' motor imagery EEG analysis in BCI-FES rehabilitation training'. Journal of Neuroscience Methods. 2013.

[37] Lydakis A et al. 'A learning-based agent for home neurorehabilitation'. International Conference on Rehabilitation Robotics. 2017.

[38] Mao Y et al. 'From assistance towards restoration with epidural brain-computer interfacing'. Conf Proc IEEE Eng Med Biol Soc. 2013.

[39] Muñoz JE, Ríos LH. Henao OA. 'Low cost implementation of a Motor Imagery experiment with BCI system and its use in neurorehabilitation'. Conf Proc IEEE Eng Med Biol Soc. 2014.

[40] Olier I, Amengual J, Vellido A. 'A variational Bayesian approach for the robust analysis of the cortical silent period from EMG recordings of brain stroke patients'. Neurocomputing. 2011; 74(1)1301-1314.

[41] Otten P, Kim J, Son SH. 'A framework to automate assessment of upper-limb motor function impairment: a feasibility study'. Sensors. 2015; 15(1):20097-20114.

[42] Parnandi A, Wade E, Matari M. 'Motor function assessment using wearable inertial sensors'. 32nd Annual International Conference of the IEEE EMBS. 2010.

[43] Pereira M, Sobolewski A, Millan JR. 'Modulation of the interhemispheric asymmetry of motor-related brain activity using brain-computer interfaces'. Conf Proc IEEE Eng Med Biol Soc. 2015; 2319-2322.

[44] Ranganathan R. 'Reorganization of finger coordination patterns through motor exploration in individuals after stroke'. Journal of NeuroEngineering and Rehabilitation. 2017; 14:90.

[45] Rouhollahi K et al. 'Design of robust adaptive controller and feedback error learning for rehabilitation in Parkinson's disease: a simulation study'. Institution of Engineering and Technology. 2016.

[46] Scano A. et al. 'Effect of human-robot interaction on muscular synergies on healthy people and post-stroke chronic patients'. International Conference on Rehabilitation Robotics. 2017.

[47] Sharma $G$ et al. 'Using an artificial neural bypass to restore cortical control of rhythmic movements in a human with quadriplegia'. Scientific Reports. 2016.

[48] Wang $X$ et al. 'Gait assessment system based on novel gait variability measures'. International Conference on Rehabilitation Robotics. 2017.

[49] Wolbrecht ET et al. 'Optimizing compliant, model-based robotic assistance to promote neurorehabilitation'. IEEE Transactions on Neural Systems and Rehabilitation Engineering. 2008; 16(3): 286-297.

[50] Yilmaz $\mathrm{O}$ et al. 'Movement related cortical potentials change after EEG-BMI rehabilitation in chronic stroke'. 6th Annual International IEEE EMBS Conference on Neural Engineering. 2013.

[51] Zich $\mathrm{C}$ et al. 'Wireless EEG with individualized channel layout enables efficient motor imagery training'. Journal of Biomedical Informatics. 2014; 126(4):698-710. 